\title{
Rethinking the Role of Demographers in Times of Crisis
}

\author{
Thoai Ngo \\ Population Council \\ Stephanie Psaki \\ Population Council
}

Follow this and additional works at: https://knowledgecommons.popcouncil.org/series_pdr_essays-covid How does access to this work benefit you? Let us know!

\section{Recommended Citation}

Ngo, Thoai D. and Stephanie R. Psaki. "Rethinking the Role of Demographers in Times of Crisis." In Covid-19 and the Global Demographic Research Agenda, edited by Landis MacKellar and Rachel Friedman, 42-46. New York: Population Council, 2021. 


\title{
Rethinking the Role of Demographers in Times of Crisis
}

\author{
THOAI D. NGO \\ STEPHANIE R. PSAKI
}

CRISES HAVE A WAY of casting light on systemic failures in our society that, although not new, were previously overlooked by those who were not directly affected. The speed with which SARS-CoV-2 has spread across the globe, leading to nearly 90 million confirmed cases of Covid-19 and 2.0 million deaths to date (Dong et al. 2020), along with devastating economic effects for millions, was largely unfathomable a year ago, even within the global health community.

Many had warned of the threat that zoonoses pose to human populations (Lederberg 1988), and recent outbreaks, including from the Zika and Ebola viruses as well as previous coronaviruses, should have served as glaring warnings. The scale of damage from Covid-19 in many countries is also perhaps not surprising, given the lack of sufficient social safety nets to protect marginalized groups. In the United States, entrenched systems of oppression and racism reinforce social and economic disparities. These include the lack of health insurance, paid leave, affordable childcare, unemployment insurance for many, and quality housing and education, which, in turn, exacerbate health outcomes among poor communities and people of color (Ngo 2020).

That is to say, in many ways this pandemic should not be a surprise, and as demographers-many with expertise in health-we should have been more prepared to respond. Some scientific disciplines have initiated swift and effective responses. Most impressively, multiple SARS-CoV-2 vaccines with high levels of efficacy have been developed in less than a year and rollout has begun. This represents a dramatic_and previously unthinkable-advance in the field of vaccine development, due in part to unprecedented collaboration. But the response from many demographers-trained in the distribution and movement of populations, a perspective that is critical to understanding disease spread-has been slow. While the Covid-19 crisis is particularly acute, it is not the only area where our discipline has been slow to respond.

Thoai D. Ngo, Stephanie R. Psaki, Population Council. 
The world has changed rapidly in recent decades, and this moment calls on demographers to ask how we can evolve with it.

While there will undoubtedly be discussions in the coming years about new topics of focus for demographers, we propose several opportunities to change how we do our work to improve our ability to shape social and health policies while being prepared to respond to the repercussions of the Covid-19 pandemic and future crises.

1. Promote research transparency and improve efficiency. During the Covid-19 pandemic, we have witnessed widespread disinformation and growing distrust for science, especially in the United States, stemming from the growing politicization of science and medical guidance. Moving forward, scientists across diverse disciplines must come together to counter this growing distrust through greater transparency. Scientists engaged in Covid-19 research have begun this process by sharing protocols for vaccine trials publicly (Doshi 2020), as well preprints of new research (Dan et al. 2020) and datasets providing information on SARS-CoV-2 and Covid-19 (Abuya et al. 2020), cultivating trust and supporting more rapid innovation in the field. Sharing our work more transparently (including data, code, and protocols) and making better use of existing data will also allow scientists to conduct critical research through a multisectoral lens and will support better collaboration across disciplines. For example, researchers who study health and poverty in urban slums can collaborate with infectious disease modelers to study contact patterns and SARS-CoV-2 transmission risks by demographic factors, including socioeconomic status (Quaife et al. 2020). Since in-person research activities have been halted or minimized in many settings due to physical distancing policies, and remote data collection comes with many methodological challenges, sharing datasets will also allow early career researchers to continue building their skills and contributions to the field.

2. Collaborate across disciplines. There are many perspectives that demographic research can offer to better understand the effects of the pandemic, anticipate risks to certain groups, and forecast needs and challenges as we rebuild. For example, social demography can be used to examine the broader social effects of Covid-19, such as on time use within households, and decisions about child marriage. Demographic modeling can predict the indirect impacts of Covid-19, including physical distancing and school closures, on the future, on issues such as migration, fertility, education, marriage, family composition, and income. These questions will be answered most effectively if demographers proactively foster collaborations within our field and form strategic ongoing partnerships between public health researchers, epidemiologists, economists, and others. As part of these collaborations, demographers 
can link different types of data to traditional population-based surveys, such as social media, spatial, and administrative data.

3. Invest in global collaborating research centers anchored by cohort studies. Ongoing community-based cohort studies offer enormous value to the demographic research field but are expensive to build and maintain. In addition to supporting collaboration across disciplines, these sites should be locally led while harnessing expertise from across the globe. Such cohorts can be used as disease surveillance systems, allowing researchers to study population movements and dynamics, examine rising social movements, and anticipate growing risks. When an outbreak occurs, scientists can easily pivot to study disease transmission, assess the health, social, and economic effects, and model the potential consequences in the larger population. These ongoing collaborative cohorts can serve as "labs" for the development of innovative solutions, including pharmaceutical as well as economic and behavioral interventions. The Rakai Community Cohort Study in Uganda (Rakai Health Sciences Program 2020), established in 1988 in response to the HIV pandemic, has been a research "lab" that produces important social and clinical innovations. If done properly, this model would save time and resources needed to establish study populations when a crisis occurs.

4. Collect data that accurately represent the challenges facing overlooked populations. Large-scale surveys, such as the Demographic and Health Surveys (DHS 2020), are nationally representative and play an invaluable role for demographers and public health researchers alike. But national surveys are rarely able to capture the conditions and characteristics of important subgroups such as indigenous populations, migrants, racial/ ethnic minorities, or gender minority groups in a specific geographic area. SARS-CoV-2 infects and kills these groups at higher rates (CDC 2020) and they often bear the biggest social and economic burden due to systematic marginalization. If surveys fail to include them, we will be perpetually ill-equipped to respond to these challenges. Similarly, urban centers are growing and becoming more complex. We will need to change the way we collect and analyze data to adequately capture the experiences of populations living in different neighborhoods within urban areas in order to advocate for more inclusive policies and interventions.

5. Find ways to quickly and effectively communicate findings with decisionmakers. A crisis like the Covid-19 pandemic underscores how essential it is to synthesize and share evidence with decision-makers swiftly and rigorously - this entire pandemic has played out more quickly than it takes to publish most peer-reviewed papers. The peer-review process 
plays an essential—although not foolproof-role in ensuring that evidence is vetted, and research methods are shared transparently. And, importantly in the midst of a crisis, the detailed methodologies shared through scientific publications allow researchers to build off one another's work. Researchers have shared preprints of papers, and many academic journals have committed to fast-tracking the publication of Covid-19-related findings, but we also need to push ourselves to find new and innovative ways of sharing findings in real time. Populations around the world are facing numerous other challenges, including climate change, urbanization, and social unrest. There is no perfect research study, even with ample time, and taking a Bayesian approach to "updating our priors" with rapidly evolving data may be more relevant and useful in times of crisis. There is often a trade-off between speed and accuracy, and research transparency should help.

This crisis presents demographers with an opportunity to ask important questions about the future of this field. What is the role of demographers in this moment? And how can that role evolve to provide relevant real-time guidance to decision-makers? The Covid-19 pandemic has both exacerbated and shed light on deep inequalities and failures in our system. As we look to the future, many have expressed the hope that we can rethink our systems to "build back better." In the same way, this global crisis has tested the ability of demographers to use their skills in support of better population health. We now have the opportunity to reflect on how we might strengthen our field to better respond to future crises.

\section{References}

Abuya, Timothy, Karen Austrian, Adan Isaac, Beth Kangwana, Faith Mbushi, Eva Muluve, Daniel Mwanga, et al. 2020. "Experiences among adults and adolescents during the COVID-19 pandemic from four locations across Kenya," Harvard Dataverse. https://doi. org/10.7910/DVN/VO7SUO.

Centers for Disease Control and Prevention (CDC). 2020. "COVID-19 hospitalization and death by race/ethnicity." November 30, 2020. https://www.cdc.gov/coronavirus/2019-ncov/ covid-data/investigations-discovery/hospitalization-death-by-race-ethnicity.html.

Dan, Jennifer M., Jose Mateus, Yu Kato, Kathryn M. Hastie, Esther Dawen Yu, Caterina E. Faliti, Alba Grifoni, Sydney I. Ramirez, et al. 2020. "Immunological memory to SARSCoV-2 assessed for up to eight months after infection," BioRxiv, November 16, 2020. https://doi.org/10.1101/2020.11.15.383323.

DHS Program-Demographic and Health Surveys (DHS). 2020. "DHS Overview." Accessed December 1, 2020. https://www.dhsprogram.com/methodology/Survey-Types/DHS.cfm.

Dong, Ensheng, Hongru Du, and Lauren Gardner. 2020. “An interactive web-based dashboard to track COVID-19 in real time," The Lancet Infectious Diseases 20(5): 533-534. doi: 10.1016/ S1473-3099(20)30120-1. (Last accessed January 7, 2021)

Doshi, Peter. 2020. "Covid-19 vaccine trial protocols released," BMJ 371. October 21, 2020. https://doi.org/10.1136/bmj.m4058. 
Lederberg, Joshua. 1988. "Medical science, infectious disease, and the unity of humankind," JAMA 260, No. 5: 684-85. August 5, 1988. https://doi.org/10.1001/ jama.1988.03410050104039.

Ngo, Thoai D. 2020. "We need a pro-poor and pro-black response to COVID-19," Think Global Health (blog). June 10, 2020. https://www.thinkglobalhealth.org/article/we-need-propoor-and-pro-black-response-covid-19.

Quaife, Matthew, Kevin van Zandvoort, Amy Gimma, Kashvi Shah, Nicky McCreesh, Kiesha Prem, Edwine Barasa, et al. 2020. "The impact of COVID-19 control measures on social contacts and transmission in Kenyan informal settlements," MedRxiv. June 7, 2020. https://doi.org/10.1101/2020.06.06.20122689.

Rakai Health Sciences Program. 2020. "Rakai Community Cohort Study-RCCS." Accessed December 1, 2020. https://www.rhsp.org/research/rccs/rccs-overview. 